\title{
Faint Features of Galaxies revealed by the Digital Co-addition of Schmidt Plates
}

\author{
S. N. Kemp
}

\author{
Department of Pure and Applied Physics, The Queen's University of \\ Belfast, Belfast, BT7 1NN, Northern Ireland
}

\section{J. Meaburn}

Department of Physics and Astronomy, University of Manchester, Manchester, M13 9PL, England

\begin{abstract}
We have digitally co-added eight IIIa-J/GG395 UKST plates centred on the IC 4296 cluster of galaxies. The factor of $\sqrt{8}$ increase in $\mathrm{S} / \mathrm{N}$ in the co-added array enables galaxy profiles to be followed, and filamentary and diffuse features to be detected, down to $b_{J}$ surface brightnesses of $28 \mathrm{mag} \operatorname{arcsec}^{-2}$. Examples of warped disks, shells, trails, diffuse IGM and extended haloes revealed by this technique are discussed and illustrated.
\end{abstract}

\section{Introduction}

The wide fields available with Schmidt telescopes enable a very large number of galaxies to be imaged simultaneously in rich fields. The depth obtainable with sky-limited plates enables very faint features of galaxies to be registered at a higher photographic density level on the resultant plate. The relatively smooth background over the wide field is an advantage for mapping extensive low surface brightness features, compared with mosaics of CCD frames. Faint features have been discovered on single plates by optical printing on to a very high contrast film (e.g. Malin 1978). The advent of fast scanning microdensitometers, which enabled high contrast images to be produced from Schmidt plates easily, created the possibility of stacking large numbers of plates to produce very deep data arrays. Malin (1988) photographically co-added plates by successively projecting them onto a film, but this method has logistical problems. The main limiting factor in digitally co-adding plate scans has always been whether there is sufficient disk storage space available, also sufficient computing power is needed to align and add the plates in a 'reasonable' time. Marston (1988) added a $\sim 1 \mathrm{deg}^{2}$ area from 9 plates, and Hawkins (1991) added a similar area from $58 \mathrm{~J}$ plates and 61 $F$ plates. In $1988 / 9$ we digitally co-added a $4 \times 4 \mathrm{deg}^{2}$ area from 8 IIIa-J/GG395 plates centred on the IC 4296 cluster of galaxies (actual centre at RA of $13^{\mathrm{h}} 34^{\mathrm{m}}$, Dec. $-34^{\circ}$ ); each plate being scanned as four $2 \times 2 \mathrm{deg}^{2}$ areas. The eight dark sky-limited UKST plates used for this project were taken in 1986, and scanned with the Automatic Plate Measuring (APM) machine at Cambridge. The pixels in the resultant data arrays were $2 \times 2 \operatorname{arcsec}^{2}$, hence each plate produced four 
$4000^{2}$ pixel arrays. One of the data arrays was then taken as a standard and the others were aligned with it using a six-parameter transformation (allowing for shift, rotation and magnification between the standard array and the array being aligned with it). The parameters of the transformations were calculated from the positions of the centroids of stars in the arrays. The eight aligned arrays were then stacked. Here we summarise results obtained so far from our pilot project on the IC 4296 field.

\section{Results for the IC 4296 field}

The co-addition of the eight plates produces an increase of a factor $\sqrt{8}$ in signalto-noise for features on the linear part of the characteristic curve of the skylimited plates, which corresponds to about 1.1 magnitudes. The surface brightness profiles of galaxies may be followed, and diffuse emission features may be detected, down to a $b_{J}$ surface brightness level of $28 \mathrm{mag} \operatorname{arcsec}^{-2}$ in the coadded dataset. Many galaxies within the field are found to have filamentary features associated with them, or are seen to have extensive 'halo' regions.

Two edge-on disk galaxies were found to have clearly visible warps in the outer parts of their disks (Kemp \& Meaburn 1993). A high contrast image of the late-type spiral galaxy MCG $06-30-005$ is displayed in Fig. 1. The disk curves to the north on the north side of the galaxy. On the south side it first curves to the north, but fainter material can be seen beyond this curving to the south. The extent of the disk in Fig. 1 is $\approx 90 \mathrm{kpc}\left(H_{0}=50 \mathrm{~km} \mathrm{~s}^{-1} \mathrm{Mpc}^{-1}\right)$. On a normal contrast image this galaxy is barely visible due to dust lane absorption. This dust absorption could cause the apparent reversal in the warp on the south side, though this change in direction of the warp may be real (it has been seen for other galaxies e.g. for NGC 4013 by comparing the optical data of Florido et al. (1991) for the inner regions with the H I mapping of Bottema et al. (1987) of the outer regions). The warp of MCG 06-30-005 is one of the most extensive detected optically. An isophote map of it resembles closely $\mathrm{H}$ I maps of galaxies with warps, showing the depth reached by the co-added data. The S0-a galaxy MCG 05-32-039 also has a clearly discernible optical warp (Kemp \& Meaburn 1993). As warps are present only in the fainter, outer parts of disks, then the use of digitised stacked Schmidt data clearly makes such warps easier to detect and measure.

The co-added dataset has proved especially useful for the detection of low surface brightness filamentary features associated with bright galaxies. A high contrast image of the S0 galaxy MCG 05-32-053 (Kemp \& Meaburn 1993) is displayed in Fig. 2. This galaxy has an extended halo from which trails of material emanate. To the north-east of the halo a pair of trails form a looplike structure. A longer structure leads north from the northern part of the halo, then turns north-eastwards. This trail may be traced at $\geq 2 \sigma$ above background for at least $80 \mathrm{kpc}$. A lower surface brightness but equally long trail leads NNW from the north-west part of the halo, then turns NNE. To the south of the halo another trail may be seen, terminating at a small satellite galaxy 3 arcmin to the south of MCG 05-32-053. If this galaxy is moving to the south-west (in projection), then all five trails curve away from this direction of motion, possibly due to interaction with the intergalactic medium. Other such 


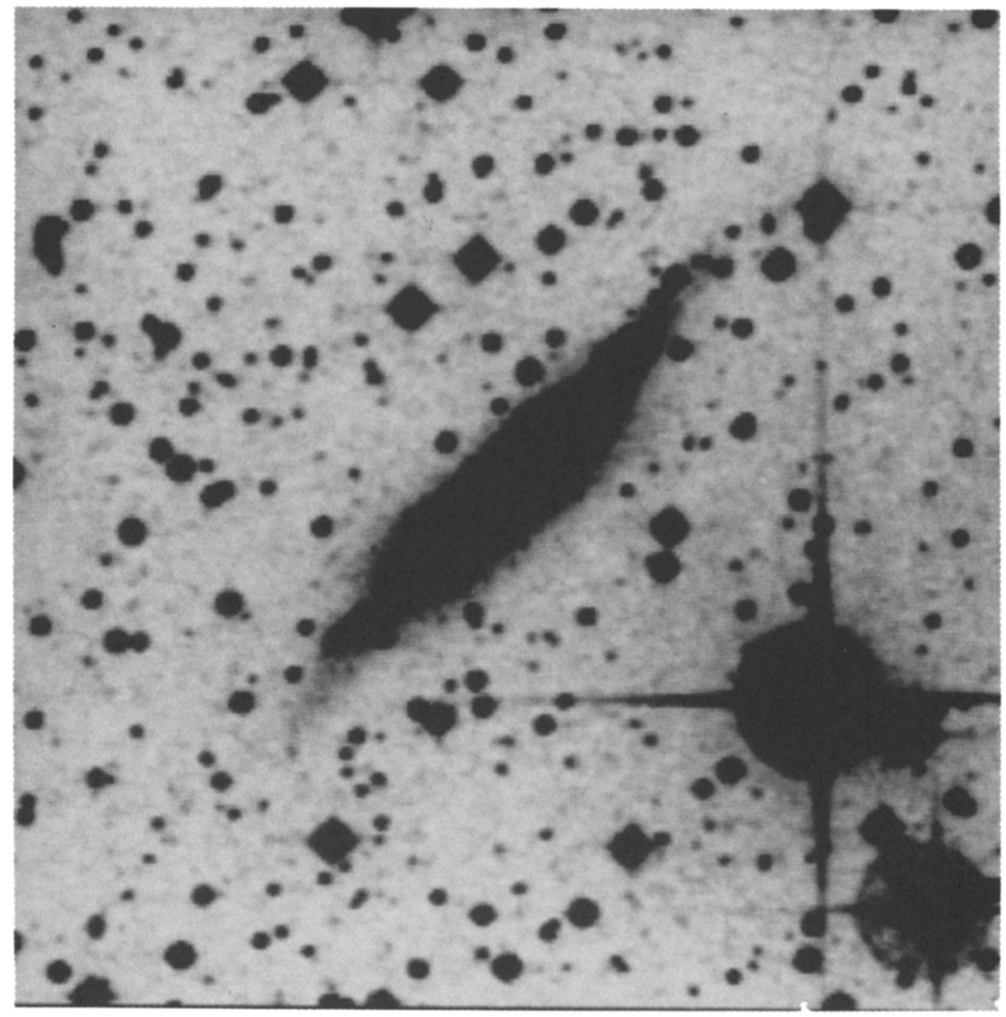

Figure 1. High contrast image of a field containing MCG 06-30-005 from the co-added array. North is to the top. The field is $8 \times 8 \mathrm{arcmin}^{2}$. The warped disk of this galaxy can be clearly seen. 


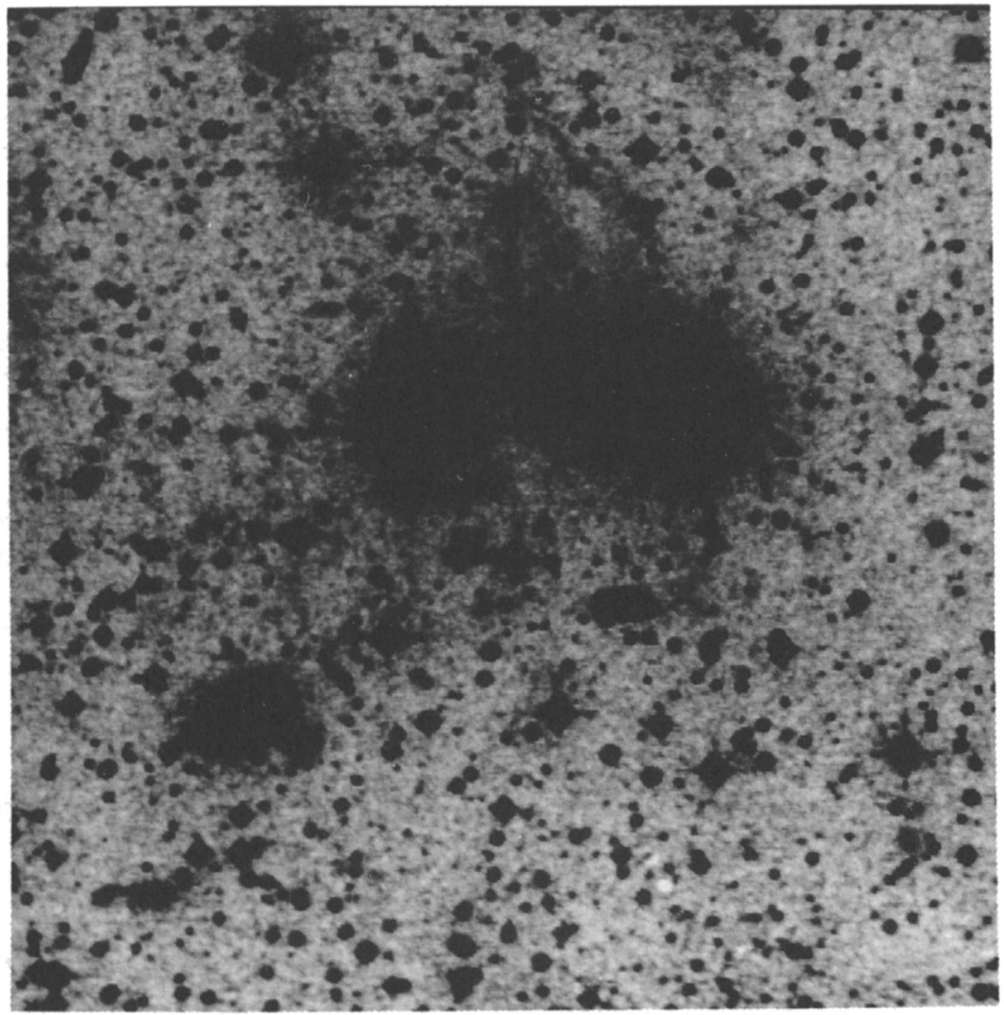

Figure 2. High contrast image of field containing MCG 05-32-053 (to the NW of the field centre) from the co-added array. North is to the top. The field is $12 \times 12 \mathrm{arcmin}^{2}$. Four trails may be seen to the $\mathrm{N}$ of the galaxy (two of which form a loop) and one to the S. 


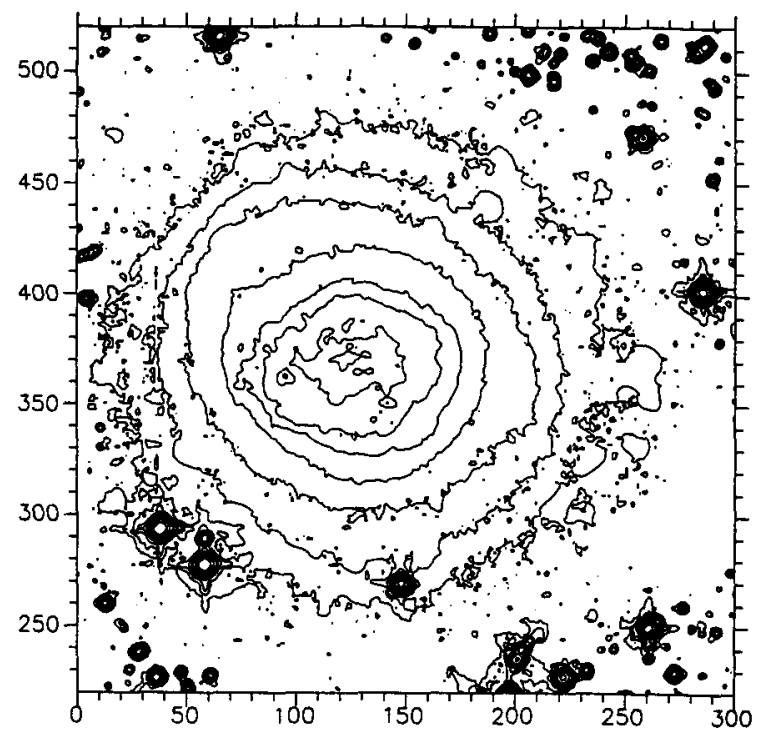

Figure 3. Plot of isodensity contours of MCG 06-30-025 from the coadded array. North is to the top. The field is $10 \times 10 \mathrm{arcmin}^{2}$. The outer contour level corresponds to a $b_{J}$ surface brightness of approximately $26.0 \mathrm{mag} \operatorname{arcsec}^{-2}$. The region of the galaxy has been interactively CLEANed of stars. The shape of the halo region is seen to be circular in projection.

faint filamentary features detected in the co-added array include a $40 \mathrm{kpc}$ long filament to the north of the pair of interacting lenticular galaxies NGC 5215a and $b$, presumably consisting of stars tidally stripped from the pair (Kemp \& Meaburn 1991a), and a shell-like feature seen adjacent to the Sdm-type galaxy MCG 05-32-063, which could be the first such feature associated with such a late-type spiral galaxy (Kemp \& Meaburn 1993). It is comparable in size and position with shells seen near elliptical galaxies (Malin \& Carter 1983), though it could possibly be a distant extension of a spiral arm seen on the other side of the galaxy. It may have been formed from the interaction of MCG 05-32-063 with a smaller spheroidal or elliptical companion.

The wide-field nature of Schmidt material may be exploited by using the coadded dataset to study the halo regions of all the galaxies in the field. This then gives very deep information on the halo structure for large samples of galaxies of each morphological type. Early-type galaxies have the most extended haloes, and this technique involving Schmidt plates has a particularly good application on such objects because of the large angular size of the field that is required (larger than most CCD imaging fields-of-view). The $\mathrm{cD}$ galaxy MCG 05-33002 is surrounded by an envelope of dimensions at least 600 by $200 \mathrm{kpc}$ (Kemp 
\& Meaburn 1991b), which becomes more elliptical with increasing radius. This envelope may consist of stars tidally stripped from cluster galaxies, or of low mass stars formed by condensation out of the X-ray cooling flow present around this galaxy (Edge \& Stewart 1991). Massive luminous baryonic haloes of this order may account for some of the 'missing' mass in clusters of galaxies, especially in connection with non-luminous mass detected in X-ray and radio haloes. The giant elliptical galaxy IC 4296 is found to have a halo of diameter at least $\sim 160 \mathrm{kpc}$ (Kemp 1994). Previous work on the isophote twisting with radius of this galaxy (e.g. Killeen et al. 1986a) has been considerably extended in radius, showing that a twist in the direction of the major axis of this galaxy of $\sim 90^{\circ}$ exists between the nuclear regions and the 'halo' (which in this case does not seem to be a separate structure). This twist orients the outer regions of the galaxy towards its near neighbour IC 4299 . IC 4296 possesses two radio jets which end in edge-brightened lobes (Killeen et al. 1986b). Faint optical emission (at the $b_{J}>27.5 \mathrm{mag} \operatorname{arcsec}^{-2}$ level) is apparent on the co-added dataset in the direction of peak radio emission of these lobes (Kemp 1994). Some latertype galaxies also have extensive haloes e.g. Fig. 3 shows an isophote map of the Magellanic-like dwarf irregular galaxy MCG 06-30-025, which is a probable member of the Cen A group of galaxies. At its outer contour level the halo of this galaxy appears circular in projection (Kemp \& Meaburn 1994). The very irregular central regions of this galaxy may be embedded in an extensive fairly spherical halo. This appears analogous to the structure of the SMC, where the older populations have a spheroidal, less centrally-concentrated distribution than the younger, main-sequence populations (Gardiner \& Hatzidimitriou 1992).

\section{Future work}

The deep, wide-field nature of the co-added dataset centred on IC 4296 has given rise to a wealth of data on the low surface brightness regions of the galaxies contained within it. While we have so far concentrated on studying galaxies and features of particular interest, there is still much work to be done, particularly statistical work on the whole galaxy sample e.g. on the galaxy luminosity functions within the dozen or so clusters contained within the field, and the compilation of a catalogue of very low surface brightness galaxies within these clusters. The question of what fraction of the luminous baryonic masses of the different galaxies resides in their halo regions, and whether this mass is sufficient to partially resolve the dark matter discrepancy, requires multicolour CCD imaging of the galaxies before it can be answered. A follow-up programme of CCD imaging, using the Anglo-Australian Telescope and the $1 \mathrm{~m}$ telescope at the South African Astronomical Observatory, has commenced, and this data will be used to calibrate the co-added array as well.

Following the success of this pilot project, the primary target for this technique must be the Virgo cluster. We have had a proposal accepted to obtain 20 original Kodak Tech Pan 4415 films, of a region of the Virgo cluster SE of its centre, over the next few years. These will be co-added, together with eight archive films with the same centre which are currently being scanned with the APM machine for this purpose. Recent tests at the UKST have shown that these films reach a magnitude or more fainter than equivalent IIIa-F plates, and galaxy 
surface brightness profiles may be followed down to $27 R$ mag $\operatorname{arcsec}^{-2}$ on a scan of a single film (Phillipps \& Parker 1993). Hence profiles in the co-added data (28 films) should be followed almost to $29 R$ mag arcsec ${ }^{-2}$. Particular priorities in the Virgo field include the investigation of whether the warping phenomenon in present is 20 prominent edge-on disk galaxies within the field; also mapping the extensive haloes of the giant elliptical galaxies within the field (including M87) and associated intracluster material.

\section{References}

Bottema R., Shostak G. S. \& van der Kruit P.C., 1987, Nature, 328, 401

Edge A. C. \& Stewart G. C., 1991, MNRAS, 252, 414

Florido E., Prieto M., Battaner E., Mediavilla E. \& Sanchez-Saavedra M. L., 1991, A\&A, 242, 301

Gardiner L. T. \& Hatzidimitriou D., 1992, MNRAS, 257, 195

Hawkins M. R. S., 1991, IAU Working Group on Wide-Field Imaging, Newsletter No. 1,23

Kemp S. N., 1994, A\&A, 282, 425

Kemp S. N., Meaburn J.. 1991a, MNRAS, 252, 27P

Kemp S. N., Meaburn J., 1991b, MNRAS, 251, 10P

Kemp S. N., Meaburn J., 1993, A\&A, 274, 19

Kemp S. N., Meaburn J., 1994, A\&A, submitted

Killeen N. E. B., Bicknell G. V. \& Carter D., 1986a, ApJ, 309, 45

Killeen N. E. B., Bicknell G. V. \& Ekers R. D., 1986b, ApJ, 302, 306

Malin D. F., 1978, Nature, 276, 591

Malin D. F., 1988, in Astrophotography, Proc. IAU , (Springer, Berlin), p. 125

Malin D. F. \& Carter D., 1983, ApJ, 274, 534

Marston, A. P. 1988, MNRAS, 230, 97

Phillipps S., Parker Q. A., 1993, MNRAS, 265, 385

\section{Discussion}

Hatzidmitriou: You mentioned that the dwarf irregular MGC 06-30-005 resembles the SMC in that it shows smooth "halo" contours and "disturbed" inner ones, indicating recent star formation there. This sort of behaviour is characteristic of dwarf irregulars in general, and can be understood in terms of threshold star formation.

Kemp: Yes, it may be true that all dwarf irregulars that are not tidally stripped by a companion galaxy may have this sort of structure (irregular core/spheroidal halo). Of course, for a "face-on" galaxy, we may just be detecting the outer parts of a "disk" structure, so no core is needed in the interpretation of the structure of any one galaxy. 